\title{
REALISASI POSITIF STABIL ASIMTOTIK SISTEM LINIER DISKRIT DENGAN POLE KONJUGAT KOMPLEKS
}

\author{
ISWAN RINA \\ Program Studi Magister Matematika, \\ Fakultas Matematika dan Ilmu Pengetahuan Alam, Universitas Andalas, \\ Kampus UNAND Limau Manis Padang, Indonesia. \\ email : mfahrinahat@yahoo.com
}

\begin{abstract}
Abstrak. Dalam artikel ini akan dikaji masalah realisasi positif stabil asimtotik dari suatu fungsi transfer yang memuat pole kompleks konjugat. Syarat perlu dan cukup untuk eksistensi realisasi positif stabil asimtotik disajikan.

Kata Kunci: Sistem linier diskrit, pole kompleks konjugat, realisasi positif stabil asimtotik
\end{abstract}

\section{Pendahuluan}

Diberikan sistem kontrol linier diskrit berikut

$$
\begin{aligned}
\mathbf{x}(t+1) & =A \mathbf{x}(t)+B \mathbf{u}(t) \\
\mathbf{y}(t) & =C \mathbf{x}(t)+D \mathbf{u}(t),
\end{aligned}
$$

dimana $A \in \mathbb{R}_{+}^{n \times n}, B \in \mathbb{R}_{+}^{n \times m}, C \in \mathbb{R}_{+}^{p \times n}$ dan $D \in \mathbb{R}_{+}^{p \times m}$. Dalam sistem (1.1), $\mathbf{x}(t) \in \mathbb{R}^{n}$ menyatakan vektor keadaan (state), $\mathbf{u}(t) \in \mathbb{R}^{m}$ menyatakan vektor input (kontrol), $\mathbf{y}(t) \in \mathbb{R}^{p}$ menyatakan vektor output, dan $t \in \mathbb{Z}_{+}$. Sistem (1.1) dikatakan positif jika $\mathbf{x}(t) \in \mathbb{R}_{+}^{n}$ dan $\mathbf{y}(t) \in \mathbb{R}_{+}^{n}$ untuk setiap kondisi awal $\mathbf{x}(0) \in \mathbb{R}_{+}^{n}$ dan semua input $\mathbf{u}(t) \in \mathbb{R}_{+}^{m}[2,5]$. Dalam [1] dinyatakan bahwa sistem [1.1] adalah positif jika dan hanya jika $A \in \mathbb{R}_{+}^{n \times n}, B \in \mathbb{R}_{+}^{n \times m}, C \in \mathbb{R}_{+}^{p \times n}$ dan $D \in \mathbb{R}_{+}^{p \times m}$. Sistem positif tersebut dikatakan stabil asimtotik jika $\lim _{t \rightarrow \infty} \mathbf{x}(t)=\mathbf{0}[2,5]$.

Fungsi transfer sistem linier diskrit merupakan perbandingan antara transformasi-z dari persamaan output dengan transformasi-z dari persamaan input dengan mengasumsikan syarat awal sama dengan nol [3]. Sehingga, fungsi transfer sistem (1.1) adalah

$$
T(z)=C\left[I_{n} z-A\right]^{-1} B+D .
$$

Dari persamaan (1.2) dapat dilihat bahwa jika persamaan (1.1) diketahui, maka fungsi transfernya dapat ditentukan dengan mudah.

Selanjutnya, misalkan $T(z)$ diberikan. Jika terdapat matriks $A \in \mathbb{R}_{+}^{n \times n}, B \in$ $\mathbb{R}_{+}^{n \times m}, C \in \mathbb{R}_{+}^{p \times n}$ dan $D \in \mathbb{R}_{+}^{p \times m}$ sedemikian sehingga

$$
T(z)=C\left[I_{n} z-A\right]^{-1} B+D,
$$


untuk matriks $A \in \mathbb{R}_{+}^{n \times n}, B \in \mathbb{R}_{+}^{n \times m}, C \in \mathbb{R}_{+}^{p \times n}$ dan $D \in \mathbb{R}_{+}^{p \times m}$ ini, jika sistem terkait yaitu

$$
\begin{aligned}
\mathbf{x}(t+1) & =A \mathbf{x}(t)+B \mathbf{u}(t) \\
\mathbf{y}(t) & =C \mathbf{x}(t)+D \mathbf{u}(t),
\end{aligned}
$$

memberikan $\mathbf{x}(t) \rightarrow \mathbf{0}$ bila $t \rightarrow \infty$ untuk setiap $\mathbf{x}(0) \in \mathbb{R}_{+}^{n}$ maka matriks $A, B, C$, dan $D$ tersebut disebut reaisasi positif stabil asimtotik dari $T(z)$.

Diberikan suatu fungsi transfer $T(z)$ orde 3 , yakni

$$
T(z)=\frac{b_{3} z^{3}+b_{2} z^{2}+b_{1} z+b_{0}}{z^{3}+a_{2} z^{2}+a_{1} z+a_{0}},
$$

yang memiliki sepasang pole konjugate kompleks dan satu pole riil, yaitu $z_{1}=\alpha$, $z_{2}=\alpha_{1}+j \beta_{1}$ dan $z_{3}=\alpha_{1}-j \beta_{1}$.

Bagaimanakah syarat yang menjamin agar terdapat matriks

$$
A \in \mathbb{R}_{+}^{n \times n}, B \in \mathbb{R}_{+}^{n \times m}, C \in \mathbb{R}_{+}^{p \times n}, D \in \mathbb{R}_{+}^{p \times m},
$$

sedemikian sehingga

$$
T(z)=C\left[I_{n} z-A\right]^{-1} B+D,
$$

dan sistem kontrol yang terkait, yaitu

$$
\begin{aligned}
\mathbf{x}(t+1) & =A \mathbf{x}(t)+B \mathbf{u}(t) \\
\mathbf{y}(t) & =C \mathbf{x}(t)+D \mathbf{u}(t),
\end{aligned}
$$

memberikan $\lim _{t \rightarrow \infty} \mathbf{x}(t)=\mathbf{0}$ untuk setiap $\mathrm{x}(0) \in \mathbb{R}_{+}^{n}$ ?

Kajian tentang masalah realisasi positif maupun realisasi positif stabil asimtotik telah dilakukan oleh berbagai peneliti. Beberapa diantaranya adalah Kitano dan Maeda [7] yang melaporkan tentang realisasi positif dari sistem kontrol linier waktu diskrit dengan pendekatan geometri, Kaczorek yang mengkaji tentang realisasi positif sistem linier waktu kontinu untuk sistem SISO [6] dan realisasi positif untuk sistem linier waktu diskrit untuk SISO [5]. Dalam makalah ini akan dikaji kembali masalah realisasi positif stabil asimtotik dari sistem linier diskrit dengan pole konjugat kompleks, dimana fungsi transfer berorde 3 .

\section{Realisasi Positif Stabil Asimtotik dari Fungsi Transfer}

Diberikan fungsi transfer sebagai berikut

$$
T(z)=\frac{b_{3} z^{3}+b_{2} z^{2}+b_{1} z+b_{0}}{z^{3}+a_{2} z^{2}+a_{1} z+a_{0}}
$$

yang mempunyai sepasang pole konjugat kompleks, dan satu pole riil.

Jika pole-pole dari persamaan (2.1) adalah $z_{1}=\alpha, z_{2}=\alpha_{1}+j \beta_{1}$, dan $z_{3}=$ $\alpha_{1}-j \beta_{1}$, maka bagian penyebut dari (2.1) dapat ditulis menjadi

$$
\begin{aligned}
d_{3}(z) & =(z-\alpha)\left(z-\alpha_{1}+j \beta_{1}\right)\left(z-\alpha_{1}-j \beta_{1}\right) \\
& =z^{3}+a_{2} z^{2}+a_{1} z+a_{0},
\end{aligned}
$$


dimana

$$
\begin{aligned}
& a_{2}=-2 \alpha_{1}-\alpha, \\
& a_{1}=\alpha_{1}^{2}+\beta_{1}^{2}+2 \alpha \alpha_{1}, \\
& a_{0}=-\alpha\left(\alpha_{1}^{2}+\beta_{1}^{2}\right) .
\end{aligned}
$$

Definisi 2.1. [2,5] Sistem (1.1) dikatakan positif jika untuk setiap $\mathbf{x}(0) \in \mathbb{R}_{+}^{n}$ dan untuk setiap $\mathbf{u}(t) \in \mathbb{R}_{+}^{m}, t \geq 0$, berlaku $\mathbf{x}(t) \in \mathbb{R}_{+}^{n}$ dan $\mathbf{y}(t) \in \mathbb{R}_{+}^{p}$.

Berdasarkan [3], solusi dari (1.1) diberikan sebagai berikut.

$$
\begin{aligned}
& \mathbf{x}(t)=A^{t} \mathbf{x}(0)+\sum_{k=0}^{t-1} A^{t-k-1} B \mathbf{u}(k), \\
& \mathbf{y}(t)=C A^{t} \mathbf{x}(0)+C \sum_{k=0}^{t-1} A^{t-k-1} B \mathbf{u}(k)+D \mathbf{u}(t), t=1,2, \cdots .
\end{aligned}
$$

Definisi 2.2. [2,5] Sistem (1.1) dikatakan stabil asimtotik jika

$$
\lim _{t \rightarrow \infty} \mathbf{x}(t)=\mathbf{0} \text { untuk setiap } \mathbf{x}(0) \in \mathbb{R}_{+}^{n} \text {. }
$$

Teorema 2.3. [2,5] Sistem (1.1) adalah positif jika dan hanya jika

$$
A \in \mathbb{R}_{+}^{n \times n}, B \in \mathbb{R}_{+}^{n \times m}, C \in \mathbb{R}_{+}^{p \times n}, D \in \mathbb{R}_{+}^{p \times m} .
$$

Teorema 2.4. [2,5] Sistem positif (1.1) adalah stabil asimtotik jika dan hanya jika semua nilai eigen $z_{1}, z_{2}, \cdots, z_{n}$ dari matriks $A \in \mathbb{R}_{+}^{n \times n}$ mempunyai modulo yang kurang dari 1, yaitu

$$
\left|z_{i}\right|<1 \text { untuk } i=1,2, \ldots, n \text {. }
$$

Lema 2.5. [1,9] Diberikan matriks A sebagai berikut

$$
A=\left[\begin{array}{lll}
a_{11} & a_{12} & a_{13} \\
a_{21} & a_{22} & a_{23} \\
a_{31} & a_{32} & a_{33}
\end{array}\right] \in \mathbb{R}_{+}^{3 \times 3} .
$$

Matriks A adalah stabil asimtotik jika dan hanya jika semua koofisien dari polinomial

$$
\bar{d}_{3}(w)=d_{3}(w+1)=w^{3}+\bar{a}_{2} w^{2}+\bar{a}_{1} w+\bar{a}_{0}
$$

dimana

$$
\begin{aligned}
& \bar{a}_{2}=3+a_{2}, \\
& \bar{a}_{1}=3+2 a_{2}+a_{1}, \\
& \bar{a}_{0}=a_{0}+a_{1}+a_{2}+1,
\end{aligned}
$$

adalah positif.

Bukti. Matriks $A$ adalah stabil asimtotik jika dan hanya jika matriks $A-I_{3} \in$ $M_{3 s}$. Selain itu $A-I_{3} \in M_{3 s}$ jika dan hanya jika koefisien-koefisien dari polinomial karakteristik adalah positif, yaitu

$$
\bar{d}_{3}(w)=w^{3}+\bar{a}_{2} w^{2}+\bar{a}_{1} w+\bar{a}_{0}
$$


dimana

$$
\begin{aligned}
\operatorname{det}\left[I_{3} w-\left(A-I_{3}\right]\right. & =\operatorname{det}\left[I_{3}(w+1)-A\right] \\
& =(w+1)^{3}+a_{2}(w+1)^{2}+a_{1}(w+1)+a_{0}, \\
& =w^{3}+\bar{a}_{2} w^{2}+\bar{a}_{1} w+\bar{a}_{0} .
\end{aligned}
$$

dimana

$$
\begin{aligned}
& \bar{a}_{2}=3+a_{2}, \\
& \bar{a}_{1}=3+2 a_{2}+a_{1}, \\
& \bar{a}_{0}=a_{0}+a_{1}+a_{2}+1 .
\end{aligned}
$$

Perhatikan matriks berikut.

$$
A_{1}=\left[\begin{array}{ccc}
a_{11} & 1 & a_{13} \\
0 & a_{22} & a_{23} \\
1 & 0 & a_{33}
\end{array}\right] \in \mathbb{R}_{+}^{3 \times 3}
$$

Polinomial karakteristik dari $A_{1}$ adalah

$$
\operatorname{det}\left[I_{3} z-A_{1}\right]=\left[\begin{array}{ccc}
z-a_{11} & -1 & -a_{13} \\
0 & z-a_{22} & -a_{23} \\
-1 & 0 & z-a_{33}
\end{array}\right]=z^{3}-a_{2} z^{2}+a_{1} z+a_{0}
$$

dimana

$$
\begin{aligned}
& a_{2}=\left(a_{11}+a_{22}+a_{33}\right) \\
& a_{1}=a_{11}\left(a_{22}+a_{33}\right)+a_{22} a_{33}-a_{13}, \\
& \left.a_{0}=-a_{11} a_{22} a_{33}\right)+a_{22} a_{13}-a_{23} .
\end{aligned}
$$

Jika $a_{0}, a_{2}, a_{1}$ diketahui dan dengan memilih $a_{11}, a_{22}, a_{33}$ sedemikian sehingga $a_{11}+$ $a_{22}+a_{33}=a_{2}$ maka dari persamaan (2.12) diperoleh

$$
\begin{aligned}
& a_{13}=a_{11}\left(a_{22}+a_{33}\right)+a_{22} a_{33}-a_{1}, \\
& \left.a_{23}=-a_{11} a_{22} a_{33}\right)+a_{22} a_{13}-a_{23} .
\end{aligned}
$$

Teorema 2.6. Terdapat realisasi positif stabil asimtotik

$$
\begin{aligned}
& \bar{A}_{k}=P A_{k} P^{-1} \in \mathbb{R}_{+}^{n \times n}, \\
& \bar{B}_{k}=P B_{k} \in \mathbb{R}_{+}^{n \times 1}, \\
& \bar{C}_{k}=C_{k} P^{-1} \in \mathbb{R}_{+}^{1 \times n}, \\
& \bar{D}_{k}=D_{k}=\left[b_{3}\right] \in \mathbb{R}_{+}, k=1,2 .
\end{aligned}
$$

untuk sebarang mariks monomial $P \in \mathbb{R}_{+}^{3 \times 3}$ dimana matriks $A_{k}, B_{k}, C_{k}, D_{k}$ berben- 
Realisasi Positif Stabil Asimtotik Sistem Linier Diskrit Dengan Pole Konjugat Kompleks 31 tuk salah satu dari yang berikut

$$
\begin{aligned}
A_{1} & =\left[\begin{array}{ccc}
a_{11} & 1 & a_{13} \\
0 & a_{22} & a_{23} \\
1 & 0 & a_{33}
\end{array}\right], \\
B_{1} & =\left[\begin{array}{c}
b_{1}+\left(a_{11}+a_{22}\right) b_{2}-\left[\left(a_{11}+a_{22}\right) a_{2}+a_{1}\right] b_{3} \\
b_{0}+a_{22} b_{1}+a_{22}^{2} b_{2}-\left(a_{0}+a_{22}^{2} a_{2}+a_{22} a_{1}\right) b_{3} \\
b_{2}-a_{2} b_{3}
\end{array}\right], \\
C_{1} & =\left[\begin{array}{lll}
0 & 0 & 1
\end{array}\right], \quad D_{1}=b_{3},
\end{aligned}
$$

atau

$$
A_{2}=A_{1}^{T}, \quad B_{2}=C_{1}^{T}, \quad C_{2}=B_{1}^{T}, \quad D_{2}=D_{1}
$$

dari fungsi transfer (2.1) jika dan hanya jika

$$
\begin{array}{r}
a_{2}^{2}-3 a_{1}>0,2 a_{2}^{3}-9 a_{1} a_{2}-27 a_{0}>0, \\
a_{2}<0,3+a_{2}>0,3+2 a_{2}+a_{1}>0, a_{0}+a_{1}+a_{2}+1>0,
\end{array}
$$

dan

$$
\begin{aligned}
b_{1}+\left(a_{11}+a_{22}\right) b_{2}-\left[\left(a_{11}+a_{22}\right) a_{2}+a_{1}\right] b_{3} & \geq 0, \\
b_{0}+a_{22} b_{1}+a_{22}^{2} b_{2}-\left(a_{0}+a_{22}^{2} a_{2}+a_{22} a_{1}\right) b_{3} & \geq 0, \\
b_{2}-a_{2} b_{3} & \geq 0 .
\end{aligned}
$$

Bukti. Berdasarkan Lema 2.5 dan (2.6), matriks $A_{1}$ adalah stabil asimtotik jika dan hanya jika syarat (2.18) terpenuhi.

Pilih $a_{11}=a_{22}=a_{33}$, maka diperoleh

$$
\begin{aligned}
& a_{11}=a_{22}=a_{33}=-\frac{a_{2}}{3}, \\
& a_{13}=3\left(\frac{a_{2}^{2}}{9}\right)^{2}-a_{1} \geq 0, \text { atau } a_{2}^{2}-3 a_{1} \geq 0 . \\
& a_{23}=2 \frac{a_{2}}{27}-\frac{a_{1} a_{2}}{3}-a_{0} \geq 0, \text { atau } 2 a_{2}^{3}-9 a_{1} a_{2}-27 a_{0}>0 .
\end{aligned}
$$

Terdapat $a_{13}>0$ dan $a_{23}>0$ jika dan hanya jika kondisi dari persamaan $(2.18)$ terpenuhi. Matriks

$$
D_{1}=\lim _{z \rightarrow \infty} T(z)=b_{3} \in \mathbb{R}_{+}^{1 \times 1}
$$

jika dan hanya jika $b_{3}>0$.

Fungsi transfer stricly proper mempunyai bentuk

$$
T_{s p}(z)=T(z)-D_{1}=\frac{\overline{b_{2}} z^{2}+\overline{b_{1}} z+\overline{b_{0}}}{z^{3}+a_{2} z^{2}+a_{1} z+a_{0}} .
$$

Jadi

$$
\begin{aligned}
T_{s p}(z) & =T(z)-D_{1} \\
& =\frac{b_{3} z^{3}+b_{2} z^{2}+b_{1} z+b_{0}}{z^{3}+a_{2} z^{2}+a_{1} z+a_{0}}-\lim _{z \rightarrow \infty} T(z), \\
& =\frac{\overline{b_{2}} z^{2}+\overline{b_{1}} z+\overline{b_{0}}}{z^{3}+a_{2} z^{2}+a_{1} z+a_{0}}
\end{aligned}
$$


dimana

$$
\overline{b_{2}}=b_{2}-a_{2} b_{3}, \overline{b_{1}}=b_{1}-a_{1} b_{3}, \overline{b_{0}}=b_{0}-a_{0} b_{3} .
$$

Dengan mengasumsikan

$$
C_{1}=\left[\begin{array}{lll}
0 & 0 & 1
\end{array}\right]
$$

diperoleh

$$
\begin{aligned}
T_{s p}(z) & =C_{1}\left[I_{3} z-A_{1}\right]^{-1} B_{1} \\
& =\left[\begin{array}{lll}
0 & 0 & 1
\end{array}\right]\left[\begin{array}{lll}
z & 0 & 0 \\
0 & z & 0 \\
0 & 0 & z
\end{array}\right]-\left[\begin{array}{ccc}
a_{11} & 1 & a_{13} \\
0 & a_{22} & a_{23} \\
1 & 0 & a_{33}
\end{array}\right]^{-1}\left[\begin{array}{l}
b_{11} \\
b_{12} \\
b_{13}
\end{array}\right] \\
& =\frac{b_{13} z^{2}\left[b_{11}-\left(a_{11}+a_{12}\right) b_{13}\right] z+b_{12}-a_{22} b_{11}+a_{11} a_{22} b_{13}}{z^{3}+a_{2} z^{2}+a_{1} z+a_{0}} .
\end{aligned}
$$

Dengan membandingkan persamaan (2.20) dengan (2.23), diperoleh

$$
\begin{aligned}
& b_{13}=\bar{b}_{2}=b_{2}-a_{2} b_{3} \\
& b_{11}=\bar{b}_{1}+\left(a_{11}+a_{22}\right) b_{13}, \\
& b_{12}=\bar{b}_{0}+a_{22} b_{11}-a_{11} a_{22} b_{13}
\end{aligned}
$$

Dari persamaan (2.15) diperoleh bahwa $B_{1} \in \mathbb{R}_{+}^{3 \times 1}$ jika dan hanya jika kondisi (2.20) terpenuhi. Berdasarkan matriks (2.14) realisasi positif stabil asimtotik untuk sebarang matriks monomial $P \in \mathbb{R}_{+}^{3 \times 3}$ jika dan hanya jika matriks dari persamaan (2.15) adalah realisasi positif stabil asimtotik.

\section{Ucapan Terima Kasih}

Penulis mengucapkan terima kasih kepada Bapak Dr. Muhafzan, Bapak Dr. Admi Nazra, Ibu Dr. Lyra Yulianti, Ibu Dr. Maiyastri, dan Ibu Dr. Yanita yang telah memberikan masukan dan saran, sehingga tulisan ini dapat diselesaikan dengan baik.

\section{Daftar Pustaka}

[1] Anton, H. 1991. Aljabar Linier Elementer. Edisi Kedelapan. Jilid 1. Erlangga, Jakarta.

[2] Farina, L and Rinaldi, S. 2000. Positive Linear Systems. John Wiley and Sons, New York.

[3] Hendricks, E, Jannerup, O dan Sensen, P. H. 2008. Linear System Control. Springer, Verlag Berlin Heidelberg.

[4] Kaczorek, T. 1991. Linear Control System. Vol. 1. Research Studies Press, England.

[5] Kaczorek, T. 2002. Positive 1D and 2D Systems. Springer-Verlag, London.

[6] Kaczorek, T. 2012. Positive Stable Realizations of Discrete-time Linear System. Buletin of the Polish Academy of Sciences, Vol. 60, No. 3

[7] Kitano, T, dan Maeda, H. 1998. Positive Realization of Discrete-Time Systems by Geometric Approach. IEEE Transactions on Circuits and Systems. Vol.45 (3): $308-311$ 
Realisasi Positif Stabil Asimtotik Sistem Linier Diskrit Dengan Pole Konjugat Kompleks 33

[8] Kwakernaak, H. 1972. Linear Optimal Control System. John Wiley and Son, Inc. Canada.

[9] Ogata, K. 1995. Discrete-Time Control Systems. Prentice Hall, New Jersey. 